\title{
Thermal vector potential theory of transport induced by temperature gradient
}

\author{
Gen Tatara \\ RIKEN Center for Emergent Matter Science (CEMS) \\ 2-1 Hirosawa, Wako, Saitama 351-0198, Japan
}

(Dated: September 19, 2018)

\begin{abstract}
A microscopic formalism to calculate thermal transport coefficients is presented based on a thermal vector potential, whose time-derivative is related to a thermal force. The formalism is free from unphysical divergences reported to arise when Luttinger's formalism is applied naively, because the equilibrium ('diamagnetic') currents are treated consistently. The mathematical structure for thermal transport coefficients are shown to be identical with the electric ones if the electric charge is replaced by energy. The results indicates that the thermal vector potential couples to energy current via the minimal coupling.
\end{abstract}

Conversion of heat into electric and other currents and vice versa is of essential importance from the viewpoint of realizing devices with low energy consumption. Of recent particular interest is heat-induced spin transport in the field of spintronics, where spin current is expected to lead to novel mechanisms for information technology, and to devices with low-energy consumption due to the absence or weak Joule heating.

A hot issue in spintronics is to use magnetic insulators, which are suitable for fast magnetization switching and low-loss signal transmission. Insulators have, however, a clear disadvantage that electric current cannot be used for its manipulation. Instead, temperature gradients become the most important driving force in inducing spin transport. To study thermally-induced spin transport theoretically, a microscopic formulation is necessary for full understanding and for quantitative predictions. A microscopic description is, however, not straightforward; temperature gradients and thermal forces are macroscopic quantities arising after statistical averaging, and thus it is not obvious how to represent those effects in a microscopic quantum mechanical Hamiltonian.

In 1964, Luttinger proposed a solution [1]. To describe the effect of temperature gradient, he introduced a scalar potential $\Psi$, which he called a 'gravitational' potential, which couples to energy density of the system, $\mathcal{E}$, via an interaction Hamiltonian,

$$
H_{\mathrm{L}}=\int d^{3} r \Psi \mathcal{E}
$$

Although the microscopic origin of the potential has not been addressed, he argued that to satisfy the Einstein relation the potential adjusts itself to balance the thermal force, resulting in an identity $\nabla \Psi=\frac{\nabla T}{T}$ in the thermal equilibrium. Owing to this trick, thermal transport coefficients can be calculated by linear response theory with respect to the field $\Psi$ without introducing the temperature gradient in a microscopic Hamiltonian. Another approach, based on the Landauer-Büttiker formalism, was presented by Butcher [2].

Luttinger's method has been applied to study various thermally-induced electron transports 3 3] , magnon transport 10] and thermally-induced torque 11. It turned out, however, that naive application often leads to apparently wrong transport coefficients which diverge as $T \rightarrow 0$ [3, 4, 7]. In the case of the thermal Hall effect, the divergence was identified to be due to a wrong treatment of the equilibrium diamagnetic current induced by the applied magnetic field, and it was found that the physical Hall coefficient is obtained if one subtracts the equilibrium magnetization current before applying linear response theory [7]. A similar problem was reported recently for thermally-driven spin-transfer torques [11].

In the case of electrically-driven transport, elimination of unphysical equilibrium contribution from transport coefficients is guaranteed by $\mathrm{U}(1)$ gauge invariance, which represents charge conservation. In the presence of an electromagnetic vector potential, $\boldsymbol{A}$, the physical electric current has two components, a paramagnetic current (the first term) and a diamagnetic current (the second term), as $\boldsymbol{j}=\frac{e}{m}\langle\hat{\boldsymbol{p}}\rangle-\frac{e^{2}}{m} n_{\mathrm{e}} \boldsymbol{A}$, where $\langle\hat{\boldsymbol{p}}\rangle$ is quantum average of the momentum density operator, $n_{\mathrm{e}}$ is electron density and $e$ and $m$ are electron's charge and mass. The paramagnetic current contains an equilibrium contribution arising from all the electrons below the Fermi level, which turns out to be $\frac{e^{2}}{m} n_{\mathrm{e}} \boldsymbol{A}$. This equilibrium contribution thus cancels perfectly with the diamagnetic contribution, leaving only the contribution from excitations in the transport coefficients [12]. Obviously, a consistent treatment of the two contributions is necessary for the cancellation of equilibrium contribution and for gaugeinvariant physical results. If one uses, instead of a vector potential, a scalar potential to describe an conservative electric field, the role of the diamagnetic current is not clearly seen, and wrong results easily arise if an inconsistent treatment is employed.

From those experiences in electrically-induced transport, the divergence in the thermally-induced transport described by Luttinger's $\Psi$ is expected to be due to an incorrect treatment of the 'diamagnetic' contribution. 13. What Qin et al. 7] showed is that the 'diamagnetic' effect is consistently taken account of if divergenceless magnetization current is included. If one could construct a vector potential representation of thermal effects, the 'diamag- 
netic' effect would be treated consistently and straightforwardly, since the 'diamagnetic' current is defined by the Hamiltonian and the vector potential without ambiguity.

Temperature gradients exert a statistical force proportional to $\nabla T$, which is conservative, i.e., has no rotation component. Still, one may introduce a rotationless vector potential to describe the force. In the case of classical charged particles described by a Hamiltonian $H=\frac{(\boldsymbol{p}-e \boldsymbol{A})^{2}}{2 m}+V(\boldsymbol{r})(V$ is a scalar potential $)$, the total force is $\boldsymbol{F}=-e \frac{\partial \boldsymbol{A}}{\partial t}-\nabla V$ if $\nabla \times \boldsymbol{A}=0$. Any rotationless force can thus be represented by use of a vector potential without introducing a scalar potential. In thermally-driven transport, a thermal force proportional to $\nabla T$ is represented by a vector potential which we call the thermal vector potential.

The objective of this paper is to propose a formalism describing thermal effects by a thermal vector potential, and to demonstrate that the formalism works perfectly for a few simple cases of thermally-driven electron and energy transport, without yielding unphysical divergences. Since the 'charge' to which the temperature gradient couples is energy, the thermal vector potential couples to the energy current density operator, $\boldsymbol{j}_{\mathcal{E}}$. We first carry out a derivation of a thermal vector potential form of the interaction Hamiltonian by looking for a Hamiltonian equivalent to the Luttinger's Hamiltonian. Local thermal equilibrium is assumed. We then derive expressions for electric current and energy current by use of conservation laws, and identify the 'diamagnetic' currents. It is shown that the 'diamagnetic' currents remove unphysical equilibrium contribution to transport coefficients. The results satisfy the Wiedemann-Franz law. We shall demonstrate that the obtained expressions for the currents indicates the minimal coupling of the thermal vector potential.

It was recently demonstrated by Shitade that a gauge theory of gravity constructed imposing local space-time translation symmetry contains a vector 'gauge field' as well as a scalar potential corresponding to that of Luttinger [14]. The model was applied to describe thermal transport of non-interacting electrons and WiedemannFranz law was shown to be satisfied. The origin of local translation symmetry in the context of thermal transport was not addressed to.

In this paper, we use energy conservation law to derive a vector potential representation of thermal effects, without assuming invariance under local space-time translation. We start with rewriting the Luttinger's Hamiltonian by use of continuity equation for operators $\mathcal{E}$ and $j_{\mathcal{E}}$

$$
\dot{\mathcal{E}}=-\nabla \cdot \boldsymbol{j}_{\mathcal{E}}
$$

as

$$
H_{\mathrm{L}}(t)=\int d^{3} r \int_{-\infty}^{t} d t^{\prime} \boldsymbol{j}_{\mathcal{E}}\left(t^{\prime}\right) \cdot \nabla \Psi(\boldsymbol{r}, t)
$$

where we used Gauss's theorem assuming that no field exists at $r \rightarrow \infty$. This expression is not of the form of an interaction between a vector potential and energy current because of the time-integration. We here look for a Hamiltonian $H_{A_{T}}$ which agrees with Eq. (3) when long time average is considered, namely, $\int_{-\infty}^{\infty} d t H_{A_{T}}=$ $\int_{-\infty}^{\infty} d t H_{\mathrm{L}}(t)$. The result is

$$
H_{A_{T}} \equiv-\int d^{3} r \boldsymbol{j}_{\mathcal{E}}(\boldsymbol{r}, t) \cdot \boldsymbol{A}_{T}(t)
$$

where $\boldsymbol{A}_{T}(t) \equiv \int_{-\infty}^{t} d t^{\prime} \nabla \Psi\left(t^{\prime}\right)$ [15] is the thermal vector potential, which satisfies

$$
\partial_{t} \boldsymbol{A}_{T}(\boldsymbol{r}, t)=\nabla \Psi(\boldsymbol{r}, t)=\frac{\nabla T}{T} .
$$

The interaction Hamiltonian (4) is understood as representing the thermodynamic potential change when a static temperature gradient is applied. In fact, the rate of the change of the entropy $(\mathcal{S})$ due to an energy current is 16 .

$$
\dot{\mathcal{S}}=-\int d^{3} r \frac{1}{T} \nabla \cdot \boldsymbol{j}_{\mathcal{E}}=-\int d^{3} r \boldsymbol{j}_{\mathcal{E}} \cdot \frac{\nabla T}{T^{2}},
$$

and this entropy change modifies the thermodynamic potential, $E-T \mathcal{S}-\mu N$ ( $E$ is the internal energy and $N$ is the electron number). The effective Hamiltonian describing a DC thermal force, $H_{\mathcal{S}} \equiv-T \mathcal{S}$, is therefore (to the linear order in $\nabla T$ )

$$
H_{\mathcal{S}}=\frac{1}{T} \int d^{3} r \int_{-\infty}^{t} j_{\mathcal{E}}\left(t^{\prime}\right) d t^{\prime} \cdot \nabla T .
$$

This reduces to Eq. (3) after the replacement $\nabla \Psi \rightarrow \frac{\nabla T}{T}$.

We now apply the thermal vector potential interaction, Eq. (44), to study thermal transport and demonstrate that the formalism works perfectly. We consider free electrons with a quadratic dispersion, described by the Hamiltonian $H_{0} \equiv \int d^{3} r \mathcal{E}_{0}$, where $\mathcal{E}_{0} \equiv \frac{\hbar^{2}}{2 m}\left(\nabla c^{\dagger}\right)(\nabla c)-$ $\mu c^{\dagger} c$ is the free electron energy density, $\mu$ is the chemical potential and $c^{\dagger}$ and $c$ are creation and annihilation operators of the electron, respectively. The energy current density is derived by use of the energy conservation law, Eq. (2). For free electrons, $\nabla \cdot \boldsymbol{j}_{\mathcal{E}}^{(0)} \equiv-\frac{i}{\hbar}\left[H_{0}, \mathcal{E}_{0}(\boldsymbol{r})\right]$, and the result is 17 .

$$
\boldsymbol{j}_{\mathcal{E}}^{(0)}=\frac{i \hbar^{3}}{(2 m)^{2}}\left[\left(\nabla^{2} c^{\dagger}\right) \nabla c-\left(\nabla c^{\dagger}\right)\left(\nabla^{2} c\right)\right]-\frac{\mu}{e} \boldsymbol{j}^{(0)},
$$

where $\boldsymbol{j}^{(0)} \equiv \frac{-i e \hbar}{2 m} c^{\dagger} \stackrel{\leftrightarrow}{\nabla} c$ is the paramagnetic part of the electric current density. We focus on the uniform 
component of the current considering the case of spatially uniform temperature gradient, which reads ( $V$ is system volume)

$$
j_{\mathcal{E}, i}^{(0)}=\frac{\hbar}{m} \frac{1}{V} \sum_{\boldsymbol{k}} k_{i} \epsilon_{\boldsymbol{k}} c_{\boldsymbol{k}}^{\dagger} c_{\boldsymbol{k}}
$$

where $\boldsymbol{k}$ is a wave vector and $\epsilon_{\boldsymbol{k}} \equiv \frac{\hbar^{2} k^{2}}{2 m}-\mu$ is the energy measured from the Fermi energy.

We now apply this interaction to study thermallydriven longitudinal electron transport on a basis of diagrammatic (Green's function) formalism. Besides the interaction Hamiltonian, we need to take account of the 'diamagnetic' current contribution proportional to $\boldsymbol{A}_{T}$. We derive it by use of the charge conservation law, $\dot{\rho}+\nabla \cdot j=0$ ( $\rho$ is electric chage density) taking account of thermal vector potential. Namely, we calculate a commutator, $-\frac{i e}{\hbar}\left[H_{A_{T}}^{0}, c^{\dagger} c\right] \equiv \nabla \cdot \boldsymbol{j}^{A_{T}}$, and derive the expression for $\boldsymbol{j}^{A_{T}}$. The result of the uniform component is

$$
j_{i}^{A_{T}}=-\frac{e}{m} A_{T, j} \frac{1}{V} \sum_{\boldsymbol{k}} \gamma_{k}^{i j} c_{\boldsymbol{k}}^{\dagger} c_{\boldsymbol{k}}
$$

where

$$
\gamma_{k}^{i j} \equiv \epsilon_{\boldsymbol{k}} \delta_{i j}+\frac{\hbar^{2}}{m} k_{i} k_{j}
$$

As expected from Eq. (9), the diagrammatic calculation is carried out by a straightforward replacement of charge $e$ in the electric field driven case 12] by energy $\epsilon_{\boldsymbol{k}}$. Including the interaction with the vector potential to the linear order, the DC paramagnetic current is

$j_{i}^{(0)}=\frac{e \hbar}{m V} \sum_{\boldsymbol{k} \omega} \epsilon_{k}^{i j}\left[\dot{A}_{T, j} \frac{f^{\prime}(\omega)}{2}\left(\phi_{\boldsymbol{k} \omega}\right)^{2}-2 A_{T, j} f(\omega) \operatorname{Im}\left[\left(g_{\boldsymbol{k}, \omega}^{\mathrm{a}}\right)^{2}\right]\right.$

where $\epsilon_{k}^{i j} \equiv \frac{\hbar^{2}}{m} k_{i} k_{j} \epsilon_{\boldsymbol{k}}, \dot{\boldsymbol{A}}_{T} \equiv \frac{\partial \boldsymbol{A}_{T}}{\partial t}, \phi_{\boldsymbol{k} \omega} \equiv g_{\boldsymbol{k}, \omega}^{\mathrm{a}}-g_{\boldsymbol{k}, \omega}^{\mathrm{r}}$, $\sum_{\omega} \equiv \int \frac{d \omega}{2 \pi}$, Im denotes the imaginary part and $f(\omega) \equiv$ $\left[e^{\beta \hbar \omega}+1\right]^{-1}$ is the Fermi distribution function $(\beta \equiv$ $(\boldsymbol{k} T)^{-1}, k_{B}$ being the Boltzmann constant). The retarded and advanced Green's functions for free electron are denoted by $g_{\boldsymbol{k}, \omega}^{\mathrm{r}}=\frac{1}{\hbar \omega-\epsilon_{\boldsymbol{k}}+\frac{i \hbar}{2 \tau}}$, and $g_{\boldsymbol{k}, \omega}^{\mathrm{a}}=\left(g_{\boldsymbol{k}, \omega}^{\mathrm{r}}\right)^{*}$, where $\tau$ is the elastic lifetime. By use of $\frac{\hbar k_{j}}{m}\left(g_{\boldsymbol{k} \omega}^{\mathrm{a}}\right)^{2}=$ $\partial_{k_{j}} g_{\boldsymbol{k} \omega}^{\mathrm{a}}$ and integration by parts with respect to $\boldsymbol{k}$, we rewrite the last contribution using

$$
\sum_{\boldsymbol{k}} \epsilon_{k}^{i j}\left[\left(g_{\boldsymbol{k} \omega}^{\mathrm{a}}\right)^{2}-\left(g_{\boldsymbol{k} \omega}^{\mathrm{r}}\right)^{2}\right]=-\sum_{\boldsymbol{k}} \gamma_{k}^{i j} \phi_{\boldsymbol{k} \omega},
$$

to obtain

$$
j_{i}^{(0)}=-\frac{e \hbar}{m V} \sum_{\boldsymbol{k} \omega} \dot{A}_{T, j} \epsilon_{k}^{i j} \frac{f^{\prime}(\omega)}{2}\left(\phi_{\boldsymbol{k} \omega}\right)^{2}-j_{i}^{A_{T}}
$$

where $j_{i}^{A_{T}}=i \frac{e \hbar}{m V} A_{T, j} \sum_{\boldsymbol{k} \omega} \gamma_{k}^{i j} f(\omega) \phi_{\boldsymbol{k} \omega}$ agrees with the diamagnetic current (Eq. (10)). The equilibrium (diamagnetic) contribution is therefore eliminated from the physical thermally-induced electric current, obtaining $j_{i}=\sigma_{T} E_{T, i}$, where

$$
\boldsymbol{E}_{T} \equiv-\frac{\partial \boldsymbol{A}_{T}(\boldsymbol{r}, t)}{\partial t}=-\frac{\nabla T}{T},
$$

is the thermal field, and $\sigma_{T} \equiv$ $\frac{e \hbar^{5}}{6 m^{2} V \tau^{2}} \sum_{k \omega} k^{2} \epsilon_{\boldsymbol{k}} f^{\prime}(\omega)\left|g_{\boldsymbol{k} \omega}^{\mathrm{r}}\right|^{4} \quad$ (assuming rotational symmetry for $\boldsymbol{k}$ ). The low temperature behavior is seen by a series expansion $\left(\Phi_{k}(\omega) \equiv\left|g_{\boldsymbol{k} \omega}^{\mathrm{r}}\right|^{4}\right)$

$$
\int_{-\infty}^{\infty} d \omega f^{\prime}(\omega) \Phi_{k}(\omega)=-\Phi_{k}(0)-\frac{\pi^{2}}{6}\left(k_{B} T\right)^{2} \Phi_{k}^{\prime \prime}(0),
$$

where $O\left(T^{4}\right)$ is neglected. Since $\epsilon_{\boldsymbol{k}}=0$ on the Fermi surface, we have $\sum_{k} k^{2} \epsilon_{\boldsymbol{k}} \Phi_{k}(0)=0$ (to the leading order of $\left.\frac{\hbar}{\epsilon_{F} \tau}\right)$. We therefore see that $\sigma_{T}=O\left(T^{2}\right)$ at $T \rightarrow 0$ and the thermally-induced current vanishes at $T=0$.

We now study the thermally-driven Hall effect and show that the vector potential formulation does not lead to unphysical results like the one reported in the Luttinger's scheme [7]. We introduce the interaction with an electromagnetic vector potential, $H_{A} \equiv-\int d^{3} r \boldsymbol{A} \cdot \boldsymbol{j}$, to describe the effect of the applied magnetic field, where $\boldsymbol{j}=\boldsymbol{j}^{(0)}+\boldsymbol{j}^{A}+\boldsymbol{j}^{A_{T}}, \boldsymbol{j}^{A} \equiv-\frac{e^{2}}{m} \boldsymbol{A} c^{\dagger} c$ being the diamagnetic current of the electromagnetic origin. The electromagnetic vector potential is treated as static but has finite wave vector, since its role here is to represent a static magnetic field, $\boldsymbol{B}=\nabla \times \boldsymbol{A}$. The thermal vector potential has an infinitesimal angular frequency $(\Omega)$ and is spatially uniform. The Hall current is calculated to the lowest order, i.e., linear in both $\boldsymbol{A}$ and $\boldsymbol{A}_{T}$. It turns out that the leading contribution is linear both in the angular frequency $\Omega$ and in the wave vector $\boldsymbol{q}$.

The contributions to the paramagnetic part of the current, $\boldsymbol{j}^{(0)}$ are shown diagrammatically in Fig. 1(a)(b). ('Diamagnetic' currents shown in Fig. 1(c), vanish, since $\boldsymbol{A}$ and $\boldsymbol{A}_{T}$ carry only either finite angular frequency or finite wave vector in the present description.) The contribution of Fig. 1(a) is

$$
j_{i}^{(\mathrm{a})}=-\frac{e^{2} \hbar^{4}}{m^{3} V}\left(\nabla_{m} A_{j}\right) \dot{A}_{T, l} \sum_{\boldsymbol{k} \omega} k_{i} k_{j} \gamma_{k}^{l m} \Phi_{\boldsymbol{k} \omega}^{(\mathrm{H})},
$$

where $\Phi_{\boldsymbol{k} \omega}^{(\mathrm{H})} \equiv \operatorname{Im}\left[f^{\prime}(\omega)\left(g_{\boldsymbol{k}, \omega}^{\mathrm{r}}\right)^{2} g_{\boldsymbol{k}, \omega}^{\mathrm{a}}+\hbar f(\omega)\left(g_{\boldsymbol{k}, \omega}^{\mathrm{a}}\right)^{4}\right]$.

Because of 'diamagnetic' current due to the thermal vector potential, $\boldsymbol{j}^{A_{T}}$, we have an interaction vertex, $-\int d^{3} r \boldsymbol{A} \cdot \boldsymbol{j}^{A_{T}}$, linear in both $\boldsymbol{A}$ and $\boldsymbol{A}_{T}$. The contribution shown in Fig. 1(b) arises from this interaction vertex. It is

$$
j_{i}^{(\mathrm{b})}=\frac{e^{2} \hbar^{4}}{m^{3} V}\left(\nabla_{m} A_{j}\right) \dot{A}_{T, l} \sum_{\boldsymbol{k} \omega} k_{i} k_{m} \gamma_{k}^{j l} \Phi_{\boldsymbol{k} \omega}^{(\mathrm{H})} .
$$




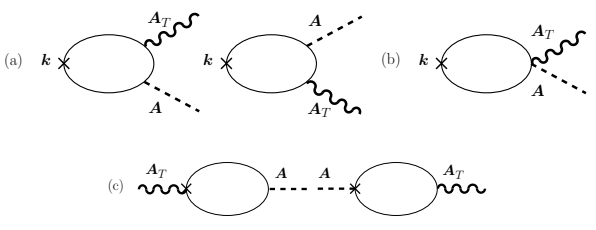

FIG. 1. Diagramatic representation of the contributions to the thermal Hall effect. Solid, wavy and dotted lines denote the electron, thermal vector potential $\boldsymbol{A}_{T}$ and the electromagnetic vector potential $\boldsymbol{A}$, respectively. Diagrams (a) and (b) correspond to contributions of Eqs. (17) and (18), respectively, while contributions of diagram (c) vanish.

The total Hall current, $j^{(\mathrm{H})} \equiv j^{(\mathrm{a})}+j^{(\mathrm{b})}$, is finally obtained as

$$
\boldsymbol{j}_{i}^{(\mathrm{H})}=\Theta_{H}\left(\boldsymbol{E}_{T} \times \boldsymbol{B}\right),
$$

where $\Theta_{H} \equiv \frac{e^{2} \hbar^{4}}{3 m^{3} V} \sum_{\boldsymbol{k} \omega} k^{2} \epsilon_{\boldsymbol{k}} \Phi_{\boldsymbol{k} \omega}^{(\mathrm{H})}$. We see that the Hall current vanishes at $T=0$ (see Eq. (16)), as is physically required. The vector potential formalism applied straightforwardly therefore leads to the correct result, in sharp contrast to Luttinger's 'gravitational' potential formalism.

For consistency of the vector potential formalism, we need to confirm that 'diagmagnetic' current arises also for the energy current. This is not a trivial issue, since we cannot invoke a gauge invariance concerning the energy current, in contrast to the case of electric current. In our scheme, 'diamagnetic' contribution is explored again by looking into the energy conservation law. In fact, including the thermal vector potential interaction, Eq. (4), in the left-hand side of Eq. (2), we see that the energy current acquires a 'diamagnetic' contribution linear in $A_{T}$. After a straightforward calculation, its uniform component is obtained as

$$
j_{\mathcal{E}, i}^{A_{T}}=-\frac{1}{m} A_{T, j} \frac{1}{V} \sum_{\boldsymbol{k}} \gamma_{T, k}^{i j} c^{\dagger}{ }_{k} c_{\boldsymbol{k}},
$$

where

$$
\gamma_{T, k}^{i j} \equiv \epsilon_{\boldsymbol{k}}\left(\epsilon_{\boldsymbol{k}} \delta_{i j}+\frac{2 \hbar^{2}}{m} k_{i} k_{j}\right) .
$$

We see here that the matrix $\gamma_{T, k}^{i j}$ for this energy current correction satisfies $\gamma_{T, k}^{i j}=\frac{\partial}{\partial k_{i}}\left[k_{j}\left(\epsilon_{\boldsymbol{k}}\right)^{2}\right]$, and thus cancellation of the unphysical equilibrium contribution occurs, in the same manner as the electric currents discussed above. The total energy current density induced by the thermal vector potential is $\boldsymbol{j}_{\mathcal{E}}=-\kappa \nabla T$, where $\kappa \equiv \frac{\hbar}{2 V T}\left(\frac{\hbar}{m}\right)^{2} \sum_{k \omega} k_{i} k_{j}\left(\epsilon_{\boldsymbol{k}}\right)^{2} f^{\prime}(\omega)\left(\phi_{\boldsymbol{k} \omega}\right)^{2}$. The coefficient satisfies the Wiedemann-Franz law, $\kappa=\frac{\pi^{2}}{3} k_{B}{ }^{2} T \sigma_{\mathrm{B}}$, where $\sigma_{\mathrm{B}}$ is the Boltzmann conductivity.

We have confirmed that thermal vector potential formalism applied for various thermally-induced transport phenomena leads straightforwardly to physical transport coefficients. The uniform contributions to the electric and energy current densities we have derived are

$$
\begin{aligned}
j_{i} & =\frac{e \hbar}{m} \frac{1}{V} \sum_{\boldsymbol{k}}\left[k_{i}-e A_{i}-\gamma_{k}^{i j} A_{T, j}\right] c_{\boldsymbol{k}}^{\dagger} c_{\boldsymbol{k}} \\
j_{\mathcal{E}, i} & =\frac{\hbar}{m} \frac{1}{V} \sum_{\boldsymbol{k}}\left[k_{i} \epsilon_{\boldsymbol{k}}-e \gamma_{k}^{i j} A_{j}-\gamma_{T, k}^{i j} A_{T, j}\right] c_{\boldsymbol{k}}^{\dagger} c_{\boldsymbol{k}} .
\end{aligned}
$$

The key for direct access to physical results in the present formalism is the particular relation between the interaction vertex and 'diamagnetic' contributions to currents, such as $\gamma_{k}^{i j}=\partial_{k_{i}}\left(k_{j} \epsilon_{\boldsymbol{k}}\right)$ and $\gamma_{T, k}^{i j}=\frac{\partial}{\partial k_{i}}\left[k_{j}\left(\epsilon_{\boldsymbol{k}}\right)^{2}\right]$. We finally show that these identities indicate that the total Hamiltonian, $H$, including the electric and thermal vector potentials are of the minimal form (to the second order in thermal vector potential) [18],

$$
H=\frac{\hbar^{2}}{2 m} \sum_{\boldsymbol{k}}\left(\boldsymbol{k}-e \boldsymbol{A}-\epsilon_{k-e A} \boldsymbol{A}_{T}\right)^{2} c_{\boldsymbol{k}}^{\dagger} c_{\boldsymbol{k}}
$$

In fact, as is easily checked, $-\frac{\delta H}{\delta A_{i}}=\frac{e \hbar}{m}\left(k_{i}-e A_{i}-\gamma_{k}^{i j} A_{T, j}\right)$ to the linear order of vector potentials $\left(\frac{\delta H}{\delta A_{i}}\right.$ denotes a functional derivative), and thus the formal definition of current, $j \equiv-\frac{\delta H}{\delta A_{i}}$, agrees with Eq. (22). As for the energy current, it is formally defined by $j_{\mathcal{E}, i} \equiv$ $-\frac{1}{2}\left(\dot{c}^{\dagger} \frac{\delta H}{\delta\left(\nabla_{i} c^{\dagger}\right)}+\frac{\delta H}{\delta\left(\nabla_{i} c\right)} \dot{c}\right)$. By use of the Heisenberg equation of motion for $\dot{c}_{k}$ and $\dot{c}_{k}^{\dagger}$, we see that this formal definition agrees with Eq. (22).

In the electromagnetic case, the minimal form is imposed by a U(1) gauge invariance. For the thermal vector potential, in contrast, there is no gauge invariance in the strict sense since the energy conservation arises from a global translational invariance with respect to time. [19] Still, our analysis indicates that the minimal form emerges. This fact might be understood as due to a 'gauge invariance' as a result of the energy conservation law. In fact, we have shown that the Luttinger's $\Psi$ and the present $\boldsymbol{A}_{T}$ have the identical effect concerning steady state properties. In other words, we may assign a part of thermal force to $\Psi$ and the rest to $\boldsymbol{A}_{T}$, so that $\frac{\nabla T}{T}=\nabla \Psi+\dot{\boldsymbol{A}}_{T}$. Thus we have a 'gauge invariance' under a transformation $\Psi \rightarrow \Psi-\dot{\chi}$ and $\boldsymbol{A}_{T} \rightarrow \boldsymbol{A}_{T}+\nabla \chi(\chi$ is a scalar function). Such a gauge transformation is generally defined for a vector field coupling to a conserved current.

The thermal vector potential formalism applies to thermal torque straightforwardly by replacing the electric charge in the derivation of Ref. [20] by energy. It is easy to check that the thermal torque vanishes at $T=0$, as is physically required.

To conclude, we have demonstrated that the calculation of transport coefficients are straightforwardly carried out based on a vector potential formalism. The formalism 
would apply straightforwardly to the case of multi-bands and to the interacting cases.

The author thanks H. Kohno, M. Ogata, H. Fukuyama, S.-K. Kim, A. Beekman, G. E. W. Bauer and N. Nagaosa for valuable comments. This work was supported by a Grant-in-Aid for Scientific Research (C) (Grant No. 25400344), (A) (Grant No. 24244053) from the Japan Society for the Promotion of Science and Grantin-Aid for Scientific Research on Innovative Areas (Grant No. 26103006) from The Ministry of Education, Culture, Sports, Science and Technology (MEXT), Japan.

[1] J. M. Luttinger, Phys. Rev. 135, A1505 (1964).

[2] P. N. Butcher, Journal of Physics: Condensed Matter 2, $4869 \quad$ (1990), URL http://stacks.iop.org/0953-8984/2/i=22/a=008.

[3] L. Smrcka and P. Streda, Journal of Physics C: Solid State Physics 10, 2153 (1977), URL http://stacks .iop.org/0022-3719/10/i=12/a=021

[4] H. Oji and P. Streda, Phys. Rev. B 31, 7291 (1985), URL http://link.aps.org/doi/10.1103/PhysRevB.31.7291

[5] G. Catelani and I. Aleiner, Journal of Experimental and Theoretical Physics 100, 331 (2005), ISSN 1063-7761, URL http://dx.doi .org/10.1134/1.1884675.

[6] K. Michaeli and A. M. Finkel'stein, Phys. Rev. B 80, 115111 (2009), URL http://link.aps.org/doi/10.1103/PhysRevB.80.115111

[7] T. Qin, Q. Niu, and J. Shi, Phys. Rev. Lett. 107, 236601 (2011), URL http://link.aps.org/doi/10.1103/PhysRevLett.107.2366 '11 $^{\circ}$

[8] G. Schwiete and A. M. Finkel'stein, Phys. Rev. B 90, 060201 (2014), URL http://link.aps.org/doi/10.1103/PhysRevB.90.060201

[9] F. G. Eich, A. Principi, M. Di Ventra, and G. Vignale, Phys. Rev. B 90, 115116 (2014), URL http://link.aps.org/doi/10.1103/PhysRevB.90.115116
[10] R. Matsumoto and S. Murakami, Phys. Rev. B 84, $184406 \quad$ (2011), URL http://link.aps.org/doi/10.1103/PhysRevB.84.184406

[11] H. Kohno, arXiv:1408.4562 (2014).

[12] J. Rammer and H. Smith, Rev. Mod. Phys. 58, 323 (1986).

[13] Since the thermal force is conservative, the 'diamagnetic'current proportional to its vector potential does not have a diamagnetic effect. We still use the term 'diagmagnetic'to denote the current contribution proportional to the vector potential.

[14] A. Shitade, Progress of Theoretical and Experimental Physics 2014, $123 \mathrm{I} 01$ (2014), http://ptep.oxfordjournals.org/content/2014/12/123I01.full.pdf+htm URL http://ptep.oxfordjournals .org/content/2014/12/123I01.

[15] We added an irrelavant constant $\int_{-\infty}^{\infty} d t^{\prime} \nabla \Psi\left(t^{\prime}\right)$ to the original form, $-\int_{t}^{\infty} d t^{\prime} \nabla \Psi\left(t^{\prime}\right)$.

[16] L. D. Landau, E. M. Lifshitz, and L. P. Pitaevskii, Electrodynamics of Continuou Media (Pergamon Press, 1984).

[17] The current determined from the continuity equation may have additional curl terms in general. Our result, however, indicates that such a term does not appear in the operator form of current in the present formalism, since the current turns out to be defined formally by the minimal coupling interaction as a sum of a paramagnetic and a 'diamagnetic' contributions. This fact is in contrast to the case of scalar potential, where the magnetization current written as a curl is essential as an equilibrium current contribution [3, 7].

[18] The minimal coupling of thermal vector potential is equivalent to the coupling between energy velocity and the vector potential in the Lagrangian representation.

] There is another difference between the thermal and the electric vector potentials: The former has no 'magnetic' component and the 'Lorentz force' for energy current does not exist.

[20] G. Tatara and P. Entel, Phys. Rev. B 78, $064429 \quad$ (pages 6) (2008), URL http://link.aps.org/abstract/PRB/v78/e064429 